\title{
One in ten patients with diabetes have suicidal thoughts after 1 year of the COVID-19 pandemic: We need to talk about diabetes and mental health not only during Suicide Prevention Awareness Month
}

\author{
Janine Alessi, ${ }^{1}\left(\mathbb{0}\right.$. Gabriela D. L. G. Scherer ${ }^{3} \cdot$ Isadora Nunes Erthal $^{3}$. Julia Belato Teixeira ${ }^{3}$. Giovana B. de Oliveira ${ }^{3}$. \\ Eduarda Herscovitz Jaeger ${ }^{3}$. Taíse Rosa de Carvalho ${ }^{2,4}$ • Beatriz D. Schaan ${ }^{1,5,6} \cdot$ Gabriela H. Telo $^{3,4,7}$
}

Received: 2 September 2021 / Accepted: 24 September 2021 / Published online: 14 October 2021

(c) Springer-Verlag Italia S.r.l., part of Springer Nature 2021

Suicide Prevention Awareness Month may have a special meaning for patients with diabetes in September 2021, almost 2 years after the onset of the COVID-19 pandemic. People living with diabetes are known to be at increased risk for mental health disorders. Compared to the general population, the prevalence of depression is two times higher among people with type 2 diabetes and three times higher among people with type 1 diabetes [1]. In usual situations, living with diabetes significantly increases the risk of suicide. The combined incidence rate of suicide in subjects with diabetes is $2.4 / 10,000$ person-years, and the proportion of long-term deaths attributable to suicide is $7.7 \%$ in type 1 diabetes and $1.3 \%$ in those with type 2 diabetes [2].

This article belongs to the topical collection Health Education and Psycho-Social Aspects, managed by Massimo Porta and Marina Trento.

Janine Alessi

janinealessi@gmail.com

1 Post-Graduate Program in Endocrinology, Universidade Federal Do Rio Grande Do Sul, Porto Alegre, Brazil

2 Endocrinology Divison, Hospital São Lucas da Pontifícia Universidade Católica do Rio Grande do Sul, Porto Alegre, Brazil

3 School of Medicine, Pontifícia Universidade Católica Do Rio Grande Do Sul, Porto Alegre, Brazil

4 Medicine and Health Sciences Graduate Program, Pontifícia Universidade Católica Do Rio Grande Do Sul, Porto Alegre, Brazil

5 School of Medicine, Universidade Federal Do Rio Grande Do Sul, Porto Alegre, Brazil

6 Endocrinology Division, Hospital de Clínicas de Porto Alegre, Porto Alegre, Brazil

7 Internal Medicine Division, Hospital São Lucas da Pontifícia Universidade Católica do Rio Grande do Sul, Porto Alegre, Brazil
Several studies have shown that the COVID-19 pandemic negatively affected mental health in people with diabetes. A study carried out by our group showed that $52.9 \%$ of patients with type 2 diabetes and $32.7 \%$ of those with type 1 diabetes had a positive screening for mental health disorders in the first 3 months of the pandemic in Brazil [3]. Diabetes-related emotional distress was also observed in $29.2 \%$ of these patients, eating disorders in $75.8 \%$, and moderate/severe sleeping disorders in $77.5 \%$ in this same period [3]. The high prevalence of mental health disorders is an alert to the risk of suicide in these patients, who may find themselves emotionally fragile during the social distancing period.

We followed a group of patients with diabetes from two university centers in Southern Brazil during the COVID19 pandemic and performed a cross-sectional analysis at the 18th month of pandemic. In total, 150 patients were included, with a mean age of $54.6 \pm 13.9$ years old and a mean diabetes duration of $21.2 \pm 10.8$ years. Mental health disorders were screened using the Self-Report Questionnaire (SRQ-20), a screening, instead of a diagnostic tool, designed to detect non-specific psychological distress, including suicidality [4]. After 18 months of pandemic, the screening for mental health disorders was positive in $46.7 \%$ of patients with type 2 diabetes and $41.4 \%$ of patients with type 1 diabetes. Alarmingly, $9.3 \%$ of participants reported thoughts about ending their own lives in the past month $(13.3 \%$ in type 2 diabetes and 5.2\% in type 1 diabetes). (Fig. 1)

In people with diabetes, the effects of the pandemic on mental health may be exacerbated by both the fear of being part of a high-risk group and because of the social distancing measures, which favor self-isolation and hinder access to health care [3]. The greater challenge in maintaining the usual diabetes care routine may result in additional concerns related to glycemic control, potentially generating more suffering for those patients. The financial repercussions of the pandemic may also lead to difficulty in obtaining supplies 


\section{$M E N T \wedge L \quad H E \wedge L T H$}

\& diabetes

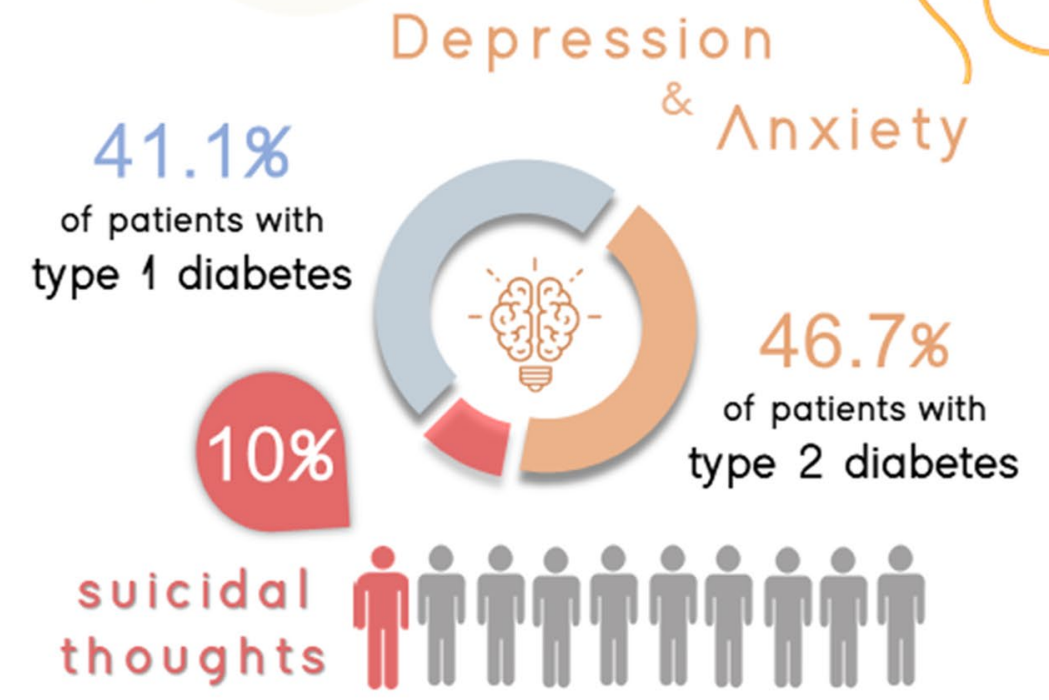

COVID-19 PANDEMIC

AND SUICIDE RISK

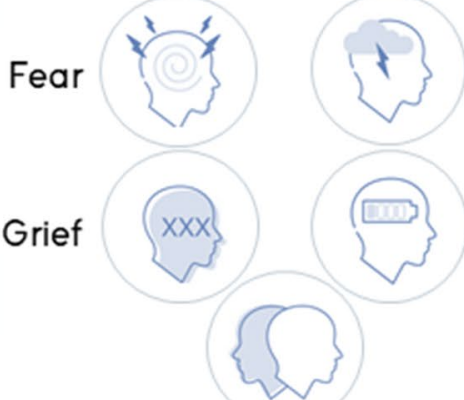

Self-isolation
Finantial

stressors

Alcohol

abuse

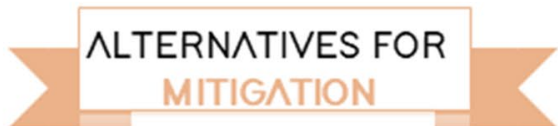

Social support

Teleconsultations

Identifying risk factors

Offering mental

health support

Fig. 1 Mental health and diabetes infographic

for the treatment of diabetes and maintaining a balanced and healthy diet, which are essential for good glycemic control.

The risk of suicide seems to be increased during the COVID-19 pandemic. People at greater risk of suicidal ideation require exceptional attention. In the insulin-treated population, the situation is even more serious, as the patient has control of a potentially lethal drug. Health professionals must be aware of the vulnerability of this group, identifying 
potential risks and offering assistance when necessary. Strategies aimed at reducing mental health disorders should be prioritized in crises [5]. The vaccination campaign appears as " $a$ light at the end of the tunnel" for the current situation. However, as long as the vaccine is not accessible to the entire population and the maintenance of distancing measures may still be necessary, we need to be attentive to identify and mitigate the risks of suicide in patients with diabetes.

Acknowledgements JA is the guarantor of this study and, as such, takes responsibility for the integrity and accuracy of the data.

Funding This work was conducted with support from Research Incentive Fund (FIPE/HCPA), Postgraduate Program in Endocrinology at the Universidade Federal do Rio Grande do Sul, School of Medical Sciences at the Pontifícia Universidade Católica do Rio Grande do Sul and Hospital São Lucas da Pontifícia Universidade Católica do Rio Grande do Sul. This study was funded in part by the Coordination for the improvement of Higher Education Personnel-Brasil (CAPES)Finance Code 001.

\section{Declarations}

Conflict of interest The authors reported no potential conflict of interest.

Ethical approval The study was carried out in accordance with the Helsinki Declaration, 2004, performed following all relevant guidelines and regulations and approved by the National Research Ethics Committee $\left(\mathrm{n}^{\circ} 4.903 .365\right)$.

Informed consent Participants provided informed consent through electronic recording (audio recording or text messaging) and all authors signed the confidentiality document for data use.

\section{References}

1. Bădescu SV, Tătaru C, Kobylinska L, Georgescu EL, Zahiu DM, Zăgrean AM, Zăgrean L. The association between Diabetes mellitus and Depression. J Med Life. 2016 Apr-Jun;9(2):120-5. PMID: 27453739 ; PMCID: PMC4863499.

2. Wang B, An X, Shi X, Zhang JA (2017) Management of endocrine disease: Suicide risk in patients with diabetes: a systematic review and meta-analysis. Eur J Endocrinol 177(4):R169-R181. https://doi.org/10.1530/EJE-16-0952 (Epub 2017 May 16 PMID: 28512134)

3. Alessi J, de Oliveira GB, Franco DW, Brino do Amaral B, Becker AS, Knijnik CP, Kobe GL, de Carvalho TR, Telo GH, Schaan BD, Telo GH. Mental health in the era of COVID-19: Prevalence of psychiatric disorders in a cohort of patients with type 1 and type 2 diabetes during the social distancing. Diabetol Metab Syndr. 2020 Aug 31;12:76. https://doi.org/10.1186/s13098-020-00584-6. PMID: 32879637; PMCID: PMC7457442.

4. Gonçalves DM, Stein AT, Kapczinski F. Avaliação de desempenho do Self-Reporting Questionnaire como instrumento de rastreamento psiquiátrico: Um estudo comparativo com o Structured Clinical Interview for DSM-IV-TR. Cad Saude Publica. 2008;

5. Alessi J, de Oliveira GB, Franco DW, Becker AS, Knijnik CP, Kobe GL, Amaral BB, de Brito A, Schaan BD, Telo GH. Telehealth strategy to mitigate the negative psychological impact of the COVID-19 pandemic on type 2 diabetes: A randomized controlled trial. Acta Diabetol. 2021 Jul;58(7):899-909. https:// doi.org/10.1007/s00592-021-01690-1. Epub 2021 Mar 15. PMID: 33723649 ; PMCID: PMC7959296.

Publisher's Note Springer Nature remains neutral with regard to jurisdictional claims in published maps and institutional affiliations. 